\title{
Oscillatory Quadratures Based on the Tau Method
}

\author{
Mohamed K. El-Daou ${ }^{1}$ \\ ${ }^{1}$ Applied Sciences Department, College of Technological Studies, Kuwait \\ Correspondence: Mohamed K. El-Daou, P.O. Box 64287 Shuwaikh/B, 70453, Kuwait. E-mail: \\ mk.eldaou@paaet.edu.kw
}

Received: April 24, 2013 Accepted: July 18, 2013 Online Published: August 8, 2013

doi:10.5539/jmr.v5n3p56 URL: http://dx.doi.org/10.5539/jmr.v5n3p56

\begin{abstract}
In this paper we discuss the integration of highly oscillatory univariate and multivariate functions. Based on the recursive formulation of the Tau method we develop numerical quadratures that achieve a high degree of accuracy when the frequency in the integrand takes moderate as well as very large values. With our procedures the integral is obtained in terms of the value of the function and its derivatives at the boundary points. The accuracy of our results are confirmed through numerical examples.
\end{abstract}

Keywords: oscillatory integral, collocation, Tau method

\section{Introduction}

Consider the integral

$$
\mathcal{I}[\omega]=\int_{a}^{b} f(x) e^{i \omega g(x)} d x
$$

where $f(x)$ and $g(x)$ are smooth functions in an interval $[a, b]$ and $\omega$ is a positive real number. By taking the real and the imaginary parts of (1) we obtain integrals with trigonometric kernels:

$$
\operatorname{Re}(\mathcal{I})=\int_{a}^{b} f(x) \cos (\omega g(x)) d x, \quad \operatorname{Im}(\mathcal{I})=\int_{a}^{b} f(x) \sin (\omega g(x)) d x .
$$

The most immediate candidate for numerically approximating integral (1) might be the standard Gauss-Christoffel quadrature (Davis \& Rabinowitz, 1980), where we interpolate the integrand at distinct nodes $c_{1}<c_{2}<\ldots<c_{v}$ in $[a, b]$ by a polynomial $p(x)$ of a prescribed degree $v-1$, and approximate

$$
\mathcal{I}[\omega] \approx \int_{a}^{b} p(x) d x
$$

When the frequency $\omega>>1$ is large, the integrand in (1) oscillates very rapidly and $\mathcal{I}[\omega]$ is called highly oscillatory. Integrals of this form arise in a wide range of science and engineering such as quantum chemistry, image analysis, acoustics, electrodynamics, computerized tomography and fluid mechanics etc. The evaluation of highly oscillatory integrals was considered as a challenging problem in the numerical analysis and computational physics. Unfortunately, if $\omega>>1$ the accuracy of approximation (2) obtained by the standard quadrature deteriorates rapidly due to the presence of sharp variations throughout $[a, b]$. The reason behind this deterioration lies in that such methods fail to detect the sharp oscillations exhibited by the integrand unless the degree of its interpolant $p(x)$ grows with the frequency. The following numerical experiment shows that while the exact value of the highly oscillatory integral decays like $O\left(\omega^{-1}\right)$, the Gauss-Christoffel quadrature produces approximate results of $O(1)$.

Consider the integral

$$
\mathcal{I}_{0}[\omega]=\int_{0}^{1}\left(3 x^{2}+2 x+1\right) e^{i \omega\left(x^{3}+x^{2}+x\right)} d x .
$$

We approximate $\mathcal{I}_{0}[\omega]$ for frequencies $100 \leq \omega \leq 6000$ using the 20-point Gauss-Christoffel quadrature; the results are plotted in Figure 1-right. On the other hand, using integration by parts we find the exact value of $\mathcal{I}_{0}[\omega]$,

$$
\mathcal{I}_{0}[\omega]=-\frac{i\left(-1+e^{3 i \omega}\right)}{\omega}=O\left(\frac{1}{\omega}\right)
$$


which is shown in Figure 1-left for the same frequencies. It is clearly seen from Figure 1 that, when $\omega$ is large, the approximate values of $\mathcal{I}_{0}[\omega]$ are of $O(1)$ whereas the exact values of $\mathcal{I}_{0}[\omega]$ behaves as $O\left(\omega^{-1}\right)$.
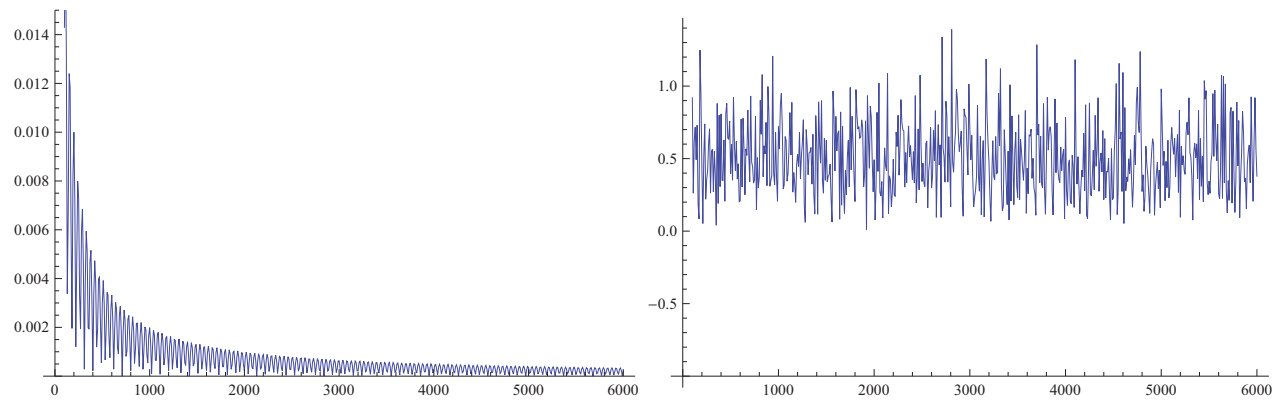

Figure 1. [left] Plot of $\left(\omega, \mathcal{I}_{0}[\omega]\right)$ where $\mathcal{I}_{0}[\omega]$ given by (3) is obtained by integration by parts. [right] Plot of $\left(\omega, \tilde{\mathcal{I}}_{0}[\omega]\right)$ where $\tilde{\mathcal{I}}_{0}[\omega] \approx \mathcal{I}_{0}[\omega]$ using the 20-point Gauss-Christoffel quadrature

This test suggests that any numerical method of a practical value must be at least of order $O\left(\omega^{-1}\right)$. The first known numerical quadrature with this order was developed in Filon (1928). Therein, Filon presented an efficient method for computing integrals of the form $(1)$ with $g(x)=x$. His approach consists of dividing the interval into $2 n$ subintervals of size $h$, and then $f(x)$ is interpolated at the endpoints and midpoint of each subinterval by a quadratic function. In each subinterval the integral becomes a polynomial multiplied by the oscillatory kernel $\sin \omega x$, which can be integrated in a closed form. This method was generalized in Luke (1954) by using higher degree polynomials in each panel, again with evenly spaced nodes. The computation of the Filon approximation rests on the ability to compute the moments

$$
\int_{a}^{b} x^{k} e^{i \omega x} d x
$$

In order to achieve a higher accuracy, Iserles and Nørsett (2005) suggested the approximation of $f(x)$ by its Hermite interpolant by choosing a sequence of nodes $\left\{x_{1}, x_{2}, \ldots, x_{v}\right\}$ associated with a sequence of multiplicities $\left\{m_{k} ; k=\right.$ $1, \ldots, v\}$, where $x_{1}=a$ and $x_{v}=b$. With this choice, and in the absence of stationary points, $(\xi$ is stationary with order $r$ if $g^{(j)}(\xi)=0$ for $j=0,1,2, \ldots, r$ but $\left.g^{(r+1)}(\xi) \neq 0\right)$, the error is of $O\left(\omega^{-s-1}\right)$ where $s:=\min \left\{m_{1}, m_{\nu}\right\}$.

Another efficient method to approximate (1) that was described in Iserles and Nørsett (2005) is the truncated asymptotic expansion of $\mathcal{I}[\omega]$ :

$$
\mathcal{I}[\omega] \sim \sum_{j=0}^{\infty} \frac{a_{j}}{\omega^{j+1}},
$$

where the coefficients $a_{j}$ depend on the function and the derivative values of $f(x)$ and $g(x)$ at the points $a$ and $b$ (Stein, 1993). The unknown coefficients $\left\{a_{j}\right\}$ depend on the moments $\int_{a}^{b} x^{k} e^{i \omega g(x)} d x$, and therefore they can be obtained explicitly if the moments are known.

Other numerical methods for approximating (1) include quadratures based on the analytic continuation and numerical steepest descent method (Huybrechs \& Vandewalle, 2006) and the exponentially fitted quadratures (Ixaru \& Vanden Berghe, 2004; Van Daele, Vanden Berghe, \& Vanden Vyver, 2005) where the weights and the nodes of the quadratures depend on the frequency of the problem.

Many of the above mentioned methods, in particular the uniform asymptotic expansion and the Filon approximation, requires an exact computation of the moments. For the particular oscillator $g(x)=x$, these moments can be obtained exactly, either through integration by parts or by using the incomplete Gamma function $\Gamma$ (Abramowitz $\&$ Stegun, 1965). However, for irregular oscillators $g(x)$ the values of the the moments

$$
\int_{a}^{b} x^{k} e^{i \omega g(x)} d x
$$

are not necessarily computable in a closed form and therefore the Filon-type and the asymptotic methods are not applicable. Thus it is necessary to find alternative techniques that are free of the moments. 
The first numerical method that approximates highly oscillatory integrals without using moments was developed in Levin (1996). Levin's approach consists in finding a function $F(x)$ such that

$$
\frac{d}{d x}\left[F(x) e^{i \omega g(x)}\right]=f(x) e^{i \omega g(x)}
$$

Expanding out the derivatives, we find that $F(x)$ satisfies the non oscillatory differential equation

$$
(D F)(x):=F^{\prime}(x)+i \omega g^{\prime}(x) F(x)=f(x)
$$

So, once $F(x)$ is obtained we immediately gain

$$
\mathcal{I}[\omega]=\int_{a}^{b} f(t) e^{i \omega g(t)} d t=F(b) e^{i \omega g(b)}-F(a) e^{i \omega g(a)} .
$$

Thus, the problem of evaluating the definite integral $\mathcal{I}[\omega]$ turns out to be a question of approximating the solution $F(x)$ of the differential Equation (4). In the absence of the stationary points, $F(x)$ is smooth and, according to Levin (1996), Equation (4) can be approximated efficiently by collocation. The latter belongs to a class of powerful techniques that use a global approach called spectral methods. In this paper we will consider solving (4) by another spectral technique called the Tau method (see Ortiz, 1969). Unlike the collocation method, the recursive nature of the Tau method permits to express the approximate value in a quadrature form, and this feature allows us to derive integration procedures competitive with the present techniques.

This paper is organized as follows: In Section 2 we recall the main features of the Tau method and we describe how to construct the Tau approximant in terms of a special polynomial basis called canonical polynomials. Section 3 is devoted to present Tau-based quadratures that estimate univariate oscillatory integrals. The case of multivariate integrals is discussed in Section 4. Numerical examples confirming our results are provided in the last section.

\section{Integration with the Tau Method}

The basic idea of the Tau method is to perturb the right hand side of Equation (4) in a way that the resulting perturbed equation can be solved analytically. More precisely, we introduce in the right hand side of (4) a perturbation term $H_{N}(x)$ such that the exact solution $F_{N}(x)$ of the equation

$$
\left(D F_{N}\right)(x):=F_{N}^{\prime}(x)+i \omega g^{\prime}(x) F_{N}(x)=f(x)+H_{N}(x)
$$

can be obtained in a closed form. Here $H_{N}(x)$ is a polynomial of degree depending on a prescribed $N \geq 1$ and whose the coefficients are adjusted in a way that $F_{N}(x)$ is found analytically. Usually $H_{N}(x)$ is chosen as a linear combination of the Chebyshev or Legendre polynomials. This is due to the fact that the equioscillatory behavior of those polynomials leads to a uniform distribution of the error throughout the interval of integration (see Ortiz, 1969). But in our context, since the main contribution to the value of $\mathcal{I}$ comes from the area of the portions neighboring the end points of $[a, b]$ (as explained in Huybrechs \& Vandewalle, 2006), it is convenient to adopt a choice for $H_{N}(x)$ that forces the differential Equation (6) and its first $N-1$ derivatives to be exact at $x=a$ and $x=b$. Of the forms that enjoys this feature is

$$
H_{N}(x)=\left(\sum_{j=0}^{r} \tau_{j}(x-a)^{j}\right)(x-a)^{N}(x-b)^{N},
$$

where $\left\{\tau_{j}, j=0,1,2, \ldots, r\right\}$ are free unknown parameters that are computed simultaneously with $F_{N}(x)$ and $r$ is fixed as explained later. Clearly (6) and (7) imply that $\left.\frac{d^{k}}{d x^{k}}\left[\left(D F_{N}\right)(x)-f(x)\right]\right|_{x \in\{a, b\}}=0$ for $k=0,1,2, \ldots, N-1$ as desired. For simplicity, let us take $a=0$ and $b=1$. Then (7) becomes

$$
H_{N}(x)=\left(\sum_{j=0}^{r} \tau_{j} x^{j}\right) x^{N}(x-1)^{N}=\sum_{j=0}^{r} \tau_{j}\left[\sum_{i=0}^{N} C_{N}^{i} x^{N+i+j}\right] ; \quad C_{N}^{i}=\frac{N !}{i !(N-i) !} .
$$

The Tau method procedure consists of expressing $F_{N}(x)$ in terms of a special polynomials basis $\left\{Q_{k}(x) ; k \geq 0\right\}$ called canonical polynomials basis. These are defined as follows: For each $k \in \mathbf{N}$, let $\widetilde{Q}_{k}$ be the exact solution of the differential equation

$$
\left(D \widetilde{Q}_{k}\right)(x)=x^{k}
$$


From Ortiz (1969), when the coefficients of the differential operator $D$ are polynomials, the set of functions $\left\{\widetilde{Q}_{k}(x) ; k \geq 0\right\}$ are generated by a self starting recursive formula. So let us consider first the case of $f(x)$ and $g^{\prime}(x)$ being polynomials of degree $\mu$ and $v$ respectively,

$$
f(x)=\sum_{j=0}^{\mu} \beta_{j} x^{j} \text { and } g^{\prime}(x)=\sum_{j=0}^{v} \alpha_{j} x^{j} .
$$

Then, for all $k \in \mathbf{N}$,

$$
D x^{k}=k x^{k-1}+i \omega \sum_{j=0}^{v} \alpha_{j} x^{j+k}=k D \widetilde{Q}_{k-1}+i \omega \sum_{j=0}^{\nu-1} \alpha_{j} D \widetilde{Q}_{j+k}+i \omega \alpha_{v} x^{\nu+k},
$$

and since $D$ is linear, we have

$$
D\left[x^{k}-k \widetilde{Q}_{k-1}-i \omega \sum_{j=0}^{v-1} \alpha_{j} \widetilde{Q}_{j+k}\right]=i \omega \alpha_{v} x^{v+k} .
$$

Comparing the latter with (9) we find that

$$
\widetilde{Q}_{v+k}(x)=\frac{1}{i \omega \alpha_{v}}\left[x^{k}-k \widetilde{Q}_{k-1}(x)-i \omega \sum_{j=0}^{v-1} \alpha_{j} \widetilde{Q}_{j+k}(x)\right], k \in \mathbf{N} .
$$

With this formula we can generate all the $\widetilde{Q}_{k}$ 's except possibly $\left\{\widetilde{Q}_{0}, \widetilde{Q}_{1}, \ldots, \widetilde{Q}_{v-1}\right\}$. For example, if $k=0$ we get

$$
\widetilde{Q}_{v}(x)=\left(\frac{1}{i \omega \alpha_{v}}\right)-\sum_{j=0}^{v-1} \frac{\alpha_{j}}{\alpha_{v}} \widetilde{Q}_{j}(x) .
$$

Therefore, each $\widetilde{Q}_{k}(x)$ can be represented as

$$
\widetilde{Q}_{k}(x)=Q_{k}(x)+\sum_{j=0}^{\nu-1} \rho_{k, j} \widetilde{Q}_{j}(x),
$$

where $\left\{Q_{k} ; k \in \mathbf{N}\right\}$ are called canonical polynomials and generated by the recursion

$$
\begin{aligned}
& Q_{k}(x)=0, \quad k=0,1,2, \ldots, v-1, \\
& Q_{v+k}(x)=\frac{1}{i \omega \alpha_{v}}\left[x^{k}-k Q_{k-1}(x)-i \omega \sum_{j=0}^{v-1} \alpha_{j} Q_{j+k}(x)\right], k \in \mathbf{N} .
\end{aligned}
$$

and $\left\{\rho_{k, l} ; k \in \mathbf{N}\right\}$ are sequences of complex numbers defined as

$$
\begin{aligned}
& \rho_{k, l}=\delta_{k}^{l} \text { (Kronecker's symbol) for } k=0,1,2, \ldots, v-1 \text { and } l=0,1,2, \ldots, v-1 \\
& \rho_{v+k, l}=\frac{1}{i \omega \alpha_{v}}\left[-k \rho_{k-1, l}-i \omega \sum_{j=0}^{v-1} \alpha_{j} \rho_{j+k, l}\right], \quad k \in \mathbf{N} \text { and } l=0,1,2, \ldots, v-1 .
\end{aligned}
$$

Now, since $D$ is linear, the exact solution of (6) can be formally expressed in terms of $\left\{\widetilde{Q}_{k}\right\}$ as follows:

$$
\begin{aligned}
F_{N}(x)= & \sum_{j=0}^{\mu} \beta_{j} \widetilde{Q}_{j}(x)+\sum_{j=0}^{r} \tau_{j}\left[\sum_{i=0}^{N} C_{N}^{i} \widetilde{Q}_{N+i+j}(x)\right] \\
= & \sum_{j=0}^{\mu} \beta_{j}\left[Q_{j}(x)+\sum_{l=0}^{v-1} \rho_{j, l} \widetilde{Q}_{l}(x)\right]+\sum_{j=0}^{r} \tau_{j}\left[\sum_{i=0}^{N} C_{N}^{i}\left[Q_{N+i+j}(x)+\sum_{l=0}^{v-1} \rho_{N+i+j, l} \widetilde{Q}_{l}(x)\right]\right] \\
= & \sum_{j=0}^{\mu} \beta_{j} Q_{j}(x)+\sum_{j=0}^{r} \tau_{j}\left[\sum_{i=0}^{N} C_{N}^{i} Q_{N+i+j}(x)\right] \\
& +\sum_{l=0}^{v-1}\left\{\sum_{j=0}^{\mu} \beta_{j} \rho_{j, l}+\sum_{j=0}^{r} \tau_{j}\left(\sum_{i=0}^{N} C_{N}^{i} \rho_{N+i+j, l}\right)\right\} \widetilde{Q}_{l}(x) .
\end{aligned}
$$


The $\left\{\tau_{k} ; k=0,1, \ldots, r\right\}$ are found then by equating the coefficients of $\left\{\widetilde{Q}_{l}(x) ; l=0,1,2, \ldots, v-1\right\}$ in (14) to 0 ,

$$
\sum_{j=0}^{r} \tau_{j}\left(\sum_{i=0}^{N} C_{N}^{i} \rho_{N+i+j, l}\right)=-\sum_{j=0}^{\mu} \beta_{j} \rho_{j, l} ; \quad l=0,1,2, \ldots, v-1 .
$$

Now if we choose $r=v-1$, Equation (15) becomes a square algebraic system consisting of $v$ equations with $v$ unknowns $\left\{\tau_{k} ; k=0,1, \ldots, v-1\right\}$, and hence the Tau method approximation $F_{N}(x)$ will be given by (13)

$$
F_{N}(x)=\sum_{j=0}^{\mu} \beta_{j} Q_{j}(x)+\sum_{j=0}^{\nu-1} \tau_{j}\left[\sum_{i=0}^{N} C_{N}^{i} Q_{N+i+j}(x)\right],
$$

which is a polynomial of degree $\leq 2 N-1$.

\section{Construction of Algorithms}

Once the approximate solution $F_{N}(x)$ to $F(x)$ is obtained as in (16), we go back to the integral (5), and write

$$
\mathcal{I}[\omega]=\int_{0}^{1} f(t) e^{i \omega g(t)} d t \approx F_{N}(1) e^{i \omega g(1)}-F_{N}(0) e^{i \omega g(0)} \equiv \mathcal{I}_{N}[\omega] .
$$

We can summarize now all the necessary steps required to compute $\mathcal{I}_{N}[\omega]$.

\subsection{Algorithm $T Q(N)$}

1) Store $\left\{\alpha_{i}\right\}_{i=0}^{v}$ and $\left\{\beta_{i}\right\}_{i=0}^{\mu}$.

2) Form $\left\{Q_{k}(x)\right\}_{k=0}^{N}$ using (11).

3) Form $\left\{\rho_{k, l} ; k=0,1,2, \ldots, N ; l=0,1,2, \ldots, v-1\right\}$ using (12).

4) Form $A_{l, j}=\sum_{i=0}^{N} C_{N}^{i} \rho_{N+i+j, l}$, for $l, j=0,1,2, \ldots, v-1$.

5) Form $B_{l}=-\sum_{j=0}^{\mu} \beta_{j} \rho_{j, l}$, for $l=0,1,2, \ldots, v-1$.

6) Compute $\left\{\tau_{j}\right\}_{j=0}^{\nu-1}$ through solving system (15): $\sum_{j=0}^{\nu-1} A_{l, j} \tau_{j}=B_{l} ; \quad l=0,1,2, \ldots, v-1$.

7) Compute $F_{N}(1)$ and $F_{N}(0)$ using (16).

8) Compute $\mathcal{I}_{N}[\omega]=F_{N}(1) e^{i \omega g(1)}-F_{N}(0) e^{i \omega g(0)}$.

If $f(x)$ and $g^{\prime}(x)$ are not polynomials, then, due to their smoothness, they can be approximated by polynomials with high degrees of accuracy. In order to be consistent with the form of $H_{N}(x)$ given in (7) we adopt the truncated two-point Taylor series expansions (see Davis, 1975):

$$
P_{2 N-1}(x)=(x-a)^{N} \sum_{k=0}^{N-1} \frac{B_{k}}{k !}(x-b)^{k}+(x-b)^{N} \sum_{k=0}^{N-1} \frac{A_{k}}{k !}(x-a)^{k},
$$

where

$$
A_{k}=\frac{d^{k}}{d x^{k}}\left[\frac{G(x)}{(x-b)^{N}}\right]_{x=a} \quad \text { and } \quad B_{k}=\frac{d^{k}}{d x^{k}}\left[\frac{G(x)}{(x-a)^{N}}\right]_{x=b},
$$

with $G(x)$ standing for $g^{\prime}(x)$ or $f(x)$. In terms of $\left\{x^{k} ; k \in \mathbf{N}\right\}$ we can write

$$
g^{\prime}(x) \approx \sum_{j=0}^{2 N-1} \alpha_{j} x^{j} \text { and } f(x) \approx \sum_{j=0}^{2 N-1} \beta_{j} x^{j}
$$

Thus we apply Algorithm TQ(N) with $\mu=v=2 N-1$.

For the special cases where $N=1$ and $N=2$, Algorithm TQ(N) takes the form of quadratures as explained next:

\subsection{Tau Quadrature $T Q(1)$}

Let $N=1, a=0$ and $b=1$. Then $\mu=v=2 N-1=1$ and (18) reduces to

$$
P_{1}(x)=x G(1)-(x-1) G(0)
$$


which gives, in accordance with (19),

$$
\begin{aligned}
g^{\prime}(x) & \approx\left(g^{\prime}(1)-g^{\prime}(0)\right) x+g^{\prime}(0) \equiv \alpha_{1} x+\alpha_{0} \quad \text { when } G=g^{\prime}, \\
f(x) & \approx(f(1)-f(0)) x+f(0) \equiv \beta_{1} x+\beta_{0} \quad \text { when } G=f
\end{aligned}
$$

where

$$
\alpha_{0}=g^{\prime}(0), \alpha_{1}=g^{\prime}(1)-g^{\prime}(0), \beta_{0}=f(0), \beta_{1}=f(1)-f(0) .
$$

The recursions (11)-(12), in turn, become

$$
\begin{aligned}
Q_{k+1}(x) & =\frac{1}{i \omega \alpha_{1}}\left[x^{k}-k Q_{k-1}-i \omega \alpha_{0} Q_{k}\right] \text { for } k \geq 0 \text { with } Q_{0}=0, \\
\rho_{k+1,0} & =\frac{1}{i \omega \alpha_{1}}\left[-k \rho_{k-1,0}-i \omega \alpha_{0} \rho_{k, 0}\right] \text { for } k \geq 0 \text { with } \rho_{0,0}=1 .
\end{aligned}
$$

In particular,

$$
\begin{gathered}
Q_{1}(x)=\frac{1}{i \omega \alpha_{1}}, \quad \rho_{1,0}=-\frac{\alpha_{0}}{\alpha_{1}}, \\
Q_{2}(x)=\frac{1}{i \omega \alpha_{1}}\left[x-\frac{\alpha_{0}}{\alpha_{1}}\right], \quad \rho_{2,0}=-\frac{1}{i \omega \alpha_{1}}+\left[\frac{\alpha_{0}}{\alpha_{1}}\right]^{2} .
\end{gathered}
$$

In this case we have $r:=v-1=0$ and therefore the Tau perturbation must contain one free parameter $\tau_{0}$ only,

$$
H_{1}(x)=\tau_{0} x(x-1)=\tau_{0}\left(x^{2}-x\right),
$$

and the linear system (15) reduces to a single equation with one unknown $\tau_{0}$,

$$
\beta_{0} \rho_{0}+\beta_{1} \rho_{1}+\tau_{0}\left(\rho_{2}-\rho_{1}\right)=0
$$

which gives

$$
\tau_{0}=\frac{\beta_{0} \rho_{0}+\beta_{1} \rho_{1}}{\rho_{1}-\rho_{2}}
$$

Substituting $\tau_{0}$ in $F_{1}(x)$ given by (16) we arrive to

$$
F_{1}(x)=\beta_{0} Q_{0}+\beta_{1} Q_{1}+\tau_{0}\left(Q_{2}-Q_{1}\right)=\left[\frac{1}{i \omega} \frac{f(1)-f(0)-i \omega f(0) g^{\prime}(1)}{g^{\prime}(1)-g^{\prime}(0)-i \omega g^{\prime}(0) g^{\prime}(1)}\right]+\left[\frac{f(0) g^{\prime}(1)-f(1) g^{\prime}(0)}{g^{\prime}(1)-g^{\prime}(0)-i \omega g^{\prime}(0) g^{\prime}(1)}\right] x .
$$

Thus the integral $\mathcal{I}[\omega]$ can be approximated by the quadrature

$$
\mathcal{I}[\omega]:=\int_{0}^{1} f(x) e^{i \omega g(x)} d x \approx F_{1}(1) e^{i \omega g(1)}-F_{1}(0) e^{i \omega g(0)}=\psi_{1} e^{i \omega g(1)}-\psi_{0} e^{i \omega g(0)} \equiv I_{1}[\omega]
$$

where

$$
\psi_{0}=\frac{1}{i \omega} \frac{f(1)-f(0)-i \omega f(0) g^{\prime}(1)}{g^{\prime}(1)-g^{\prime}(0)-i \omega g^{\prime}(0) g^{\prime}(1)} \text { and } \psi_{1}=\frac{1}{i \omega} \frac{f(1)-f(0)-i \omega f(1) g^{\prime}(0)}{g^{\prime}(1)-g^{\prime}(0)-i \omega g^{\prime}(0) g^{\prime}(1)},
$$

provided that the denominator in (21) is nonzero. It is clearly seen that quadrature TQ(1) expresses the approximate integral $\mathcal{I}_{1}[\omega]$ in terms of the values of $\left\{f(x), g^{\prime}(x)\right\}$ evaluated at the boundaries of the interval $[0,1]$. It is also important to point out that $\psi_{0}$ and $\psi_{1}$ as well as $\mathcal{I}_{1}[\omega]$ are of $O\left(\omega^{-1}\right)$ unless $g^{\prime}(0)=0$ or $g^{\prime}(1)=0$.

\subsection{Tau Quadrature $T Q(2)$}

The construction of a quadrature for $N=2$ proceeds in the same manner as above. Taking $N=2, a=0$ and $b=1$, then $\mu=v=3$ and (18)-(19) give

$$
\begin{aligned}
& f(x) \approx(x-1)^{2}\left(c_{0}+c_{1} x\right)+x^{2}\left(d_{0}+d_{1}(x-1)\right) \equiv \sum_{j=0}^{3} \alpha_{j} x^{j} \\
& g^{\prime}(x) \approx(x-1)^{2}\left(a_{0}+a_{1} x\right)+x^{2}\left(b_{0}+b_{1}(x-1)\right) \equiv \sum_{j=0}^{3} \beta_{j} x^{j}
\end{aligned}
$$


where

$$
\begin{aligned}
& a_{0}=g^{\prime}(0), \quad b_{0}=g^{\prime}(1), \quad a_{1}=2 g^{\prime}(0)+g^{\prime \prime}(0), \quad b_{1}=-2 g^{\prime}(1)+g^{\prime \prime}(1), \\
& c_{0}=f(0), \quad d_{0}=f(1), \quad c_{1}=2 f(0)+f^{\prime}(0), \quad d_{1}=-2 f(1)+f^{\prime}(1),
\end{aligned}
$$

or

$$
\begin{aligned}
& \alpha_{0}=g^{\prime}(0), \quad \alpha_{1}=g^{\prime \prime}(0), \quad \alpha_{2}=-3 g^{\prime}(0)+3 g^{\prime}(1)-2 g^{\prime \prime}(0)-g^{\prime \prime}(1), \quad \alpha_{3}=2 g^{\prime}(0)-2 g^{\prime}(1)+g^{\prime \prime}(0)+g^{\prime \prime}(1), \\
& \beta_{0}=f(0), \quad \beta_{1}=f^{\prime}(0), \quad \beta_{2}=-3 f(0)+3 f(1)-2 f^{\prime}(0)-f^{\prime}(1), \quad \beta_{3}=2 f(0)-2 f(1)+f^{\prime}(0)+f^{\prime}(1) .
\end{aligned}
$$

Since $r:=v-1=3-1=2$, we need three free parameters $\left\{\tau_{0}, \tau_{1}, \tau_{2}\right\}$,

$$
H_{3}(x)=\left(\sum_{j=0}^{2} \tau_{j} x^{j}\right) x^{3}(x-1)^{3},
$$

which are found by solving the $3 \times 3$ linear system resulting from (15). Substituting $\left\{\tau_{0}, \tau_{1}, \tau_{2}\right\}$ in $F_{3}(x)$ given by (16), we compute $F_{3}(1)$ and $F_{3}(0)$ and arrive to

$$
\mathcal{I}[\omega]:=\int_{0}^{1} f(x) e^{i \omega g(x)} d x \approx \frac{1}{D}\left(\psi_{1} e^{i \omega g(1)}-\psi_{0} e^{i \omega g(0)}\right) \approx \mathcal{I}_{2}[\omega],
$$

where

$$
\begin{aligned}
\psi_{0}= & -6\left(c_{1}+d_{1}\right)+\left(6 a_{0} c_{0}+2 b_{1} c_{0}-2 b_{0} c_{1}+b_{1} c_{1}+6 b_{0} d_{0}+2 b_{1} d_{0}-2 b_{0} d_{1}\right) i \omega \\
& +\left(2 b_{0}^{2} c_{0}-2 a_{0} b_{0} c_{0}+a_{0} b_{1} c_{0}+b_{0}^{2} c_{1}\right) \omega^{2}-a_{0} b_{0}^{2} c_{0} i \omega^{3}, \\
\psi_{1}= & -6\left(c_{1}+d_{1}\right)+\left(6 a_{0} c_{0}-2 a_{1} c_{0}+2 a_{0} c_{1}-2 a_{1} d_{0}+6 b_{0} d_{0}+2 a_{0} d_{1}+a_{1} d_{1}\right) i \omega \\
& +\left(2 a_{0} b_{0} d_{0}-2 a_{0}^{2} d_{0}+a_{1} b_{0} d_{0}+a_{0}^{2} d_{1}\right) \omega^{2}-a_{0}^{2} b_{0} d_{0} i \omega^{3}, \\
D= & -6\left(a_{1}+b_{1}\right) \omega+\left(2 a_{1} b_{0}-6 a_{0}^{2}-6 b_{0}^{2}-2 a_{0} b_{1}-a_{1} b_{1}\right) \omega^{2} \\
& +\left(2 a_{0} b_{0}^{2}-2 a_{0}^{2} b_{0}+a_{1} b_{0}^{2}+a_{0}^{2} b_{1}\right) i \omega^{3}+a_{0}^{2} b_{0}^{2} \omega^{4},
\end{aligned}
$$

provided that $D \neq 0$. Again quadrature TQ(2) expresses $\mathcal{I}_{2}[\omega]$ in terms of $\left\{f^{(i)}(0), g^{(i)}(1) ; i=0,1,2\right\}$. Here $\mathcal{I}_{2}[\omega]=O\left(\omega^{-1}\right)$ when $\left\{f^{(i)}(0), g^{(i)}(1) ; i=1,2\right\}$ are all nonzero.

Finally we point out that in order to apply our results to an arbitrary interval $[a, b]$,

$$
\int_{a}^{b} \tilde{f}(t) e^{i \omega \tilde{g}(t)} d t
$$

we simply shift $[a, b]$ to $[0,1]$ with $x=\frac{t-a}{b-a}$ and set

$$
\int_{a}^{b} \tilde{f}(t) e^{i \omega \tilde{g}(t)} d t=(b-a) \int_{0}^{1} \tilde{f}((b-a) x+a) e^{i \omega \tilde{g}((b-a) x+a)} d x=(b-a) \int_{0}^{1} f(x) e^{i \omega g(x)} d x,
$$

where $f(x)=\tilde{f}((b-a) x+a)$ and $g(x)=\tilde{g}((b-a) x+a)$.

\section{Multivariate Oscillatory Functions}

In this section we consider the integral

$$
\mathcal{I}[\omega]=\int_{\Omega} f(\mathbf{x}) e^{i \omega g(\mathbf{x})} d \mathbf{x},
$$

where $f(\mathbf{x})$ and $g(\mathbf{x})$ are smooth functions in a domain $\Omega \subset \mathbf{R}^{s}=\mathbf{R} \times \mathbf{R} \times \ldots \times \mathbf{R},(s \geq 1), d \mathbf{x}=d x_{1} d x_{2} \ldots d x_{s}$ and $\omega>>1$ is a large real number.

The integration procedure presented in the previous section can be readily extended to multivariate highly oscillatory integrals of the form (1) with $s \geq 2$, see (Olver, 2006) and (Iserles \& Nørsett, 2006). To this end we need some notations: For all $k=1,2, \ldots, s$ set

$$
\mathbf{x}_{k}:=\left(x_{k}, x_{k+1}, \ldots, x_{s}\right),
$$


and let $u_{k}$ and $v_{k}$ be functions of $\mathbf{x}_{k+1}$ :

$$
u_{k}=u_{k}\left(\mathbf{x}_{k+1}\right) \text { and } v_{k}=v_{k}\left(\mathbf{x}_{k+1}\right), \quad k=1,2, \ldots, s-1,
$$

with $u_{s} \equiv a$ and $v_{s} \equiv b$ (constants), and

$$
\mathbf{d x}_{k}:=d x_{k} d x_{k+1} \ldots d x_{s}
$$

Then (23) takes the explicit form

$$
\mathcal{I}[\omega]=\int_{u_{s}}^{v_{s}} \int_{u_{s-1}}^{v_{s-1}} \ldots \int_{u_{1}}^{v_{1}} f\left(x_{1}, x_{2}, \ldots, x_{s}\right) d x_{1} d x_{2} \ldots d x_{s}=\int_{u_{s}}^{v_{s}} \int_{u_{s-1}}^{v_{s-1}} \ldots \int_{u_{1}}^{v_{1}} f\left(\mathbf{x}_{1}\right) \mathbf{d} \mathbf{x}_{\mathbf{1}} .
$$

In order to approximate $\mathcal{I}$ we split it into a set of $s$ univariate integrals as follows:

$$
\begin{aligned}
f^{(-1)}\left(\mathbf{x}_{2}\right)= & \int_{u_{1}\left(\mathbf{x}_{2}\right)}^{v_{1}\left(\mathbf{x}_{2}\right)} f\left(\mathbf{x}_{1}\right) \mathbf{d} \mathbf{x}_{1}, \\
f^{(-2)}\left(\mathbf{x}_{3}\right)= & \int_{u_{2}\left(\mathbf{x}_{3}\right)}^{v_{2}\left(\mathbf{x}_{3}\right)} f^{(-1)}\left(\mathbf{x}_{2}\right) \mathbf{d} \mathbf{x}_{2}, \\
f^{(-k)}\left(\mathbf{x}_{k+1}\right)= & \int_{u_{k}\left(\mathbf{x}_{k+1}\right)}^{v_{k}\left(\mathbf{x}_{k+1}\right)} f^{(-k+1)}\left(\mathbf{x}_{k}\right) \mathbf{d} \mathbf{x}_{k}, \\
& \vdots \\
f^{(-s+1)}\left(\mathbf{x}_{s}\right)= & \int_{u_{s-1}\left(\mathbf{x}_{s}\right)}^{v_{s-1}\left(\mathbf{x}_{s}\right)} f^{(-s+2)}\left(\mathbf{x}_{s-1}\right) \mathbf{d} \mathbf{x}_{s-1}, \\
\mathcal{I}[\omega]= & \int_{u_{s}}^{v_{s}} f^{(-s+1)}\left(\mathbf{x}_{s}\right) \mathbf{d} \mathbf{x}_{s} .
\end{aligned}
$$

Now applying the algorithm TQ(N), developed in the previous section, to the highly oscillatory integral $f^{(-1)}(x)$ we obtain a new integral approximating $f^{(-2)}(x)$ with one dimension less. We can thus iterate the procedure on each dimension and obtain $\left\{f^{(-3)}(x), f^{(-4)}(x), \ldots, f^{(-s+1)}(x)\right\}$ eventually arriving at the univariate integral $\mathcal{I}[\omega]$ that can be approximated using the same procedure.

\section{Numerical Examples}

This section is devoted to illustrate the accuracy of our results through a set of examples.

Example 1 Let us evaluate the definite integral

$$
\mathcal{I}[\omega]=\int_{0}^{1}\left(x^{3}+4 x\right) e^{i \omega\left(x^{3}+x^{2}+x\right)} d x .
$$

The oscillatory behavior of $\left(x^{3}+4 x\right) \cos \omega\left(x^{3}+x^{2}+x\right)$, the real part of the integrand, is shown in Figure 2 for $\omega=80$. We approximated $\mathcal{I}[\omega]$ by $\mathcal{I}_{N}[\omega]$ using Algorithm TQ(N) with $N=1,2,3,4,5$ for different values of frequencies $\omega$. The errors committed by TQ(N), $\mathcal{E}_{N}[\omega]:=\mathcal{I}[\omega]-\mathcal{I}_{N}[\omega]$, are listed in Table 1 for some selected values of the frequency $\omega$. We have also plotted in Figure 2 the scaled error $\omega^{N+1} \mathcal{E}_{N}[\omega]$ for $N=1$ and $N=2$. One can easily notice that Algorithm TQ(N) achieves the asymptotic accuracy as in (Levin, 1996): $\omega^{N+1} \mathcal{E}_{N}[\omega]=O(1)$. In other words, asymptotically we have $\mathcal{E}_{N}[\omega]=O\left(\omega^{-N-1}\right)$. 


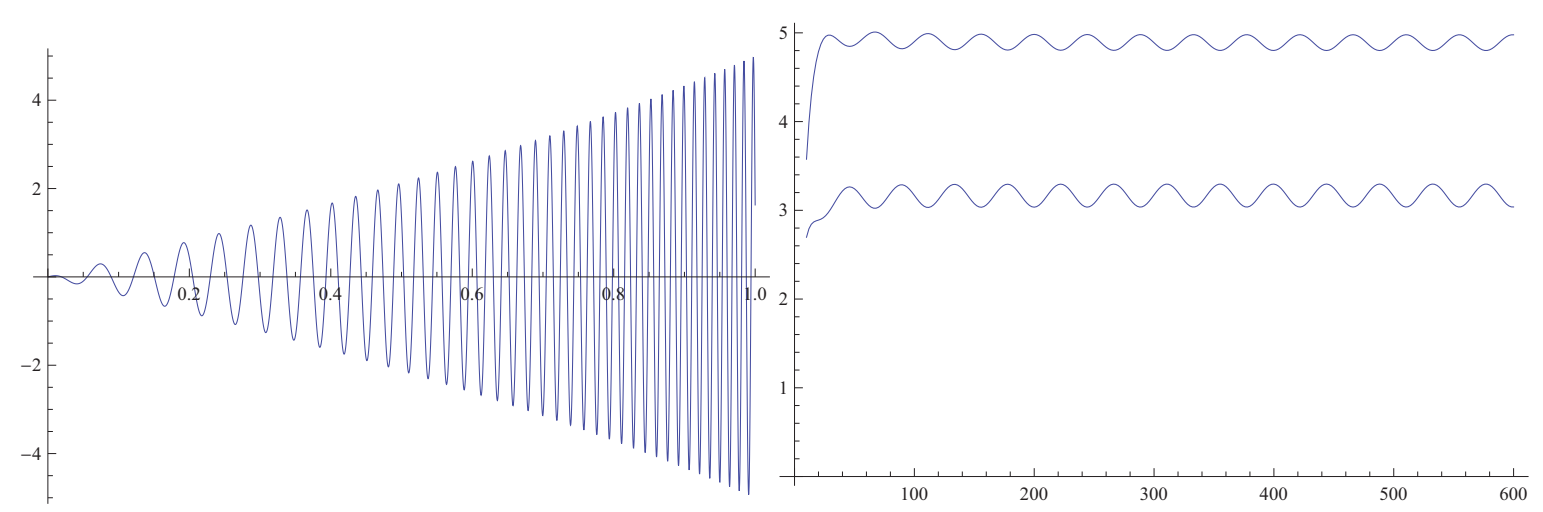

Figure 2 (Example 1). [left] Plot of $\left(x^{3}+4 x\right) \cos 80\left(x^{3}+x^{2}+x\right)$. [right] Plot of the scaled errors $\omega^{2} \mid \mathcal{E}_{1}[\omega]$ (up) and $\omega^{3}\left|\mathcal{E}_{2}[\omega]\right|($ down) where $10 \leq \omega \leq 600$

Table 1 (Example 1). Integral (24) is approximated by TQ(N), $N=1,2,3,4,5$. List of $\left|\mathcal{E}_{N}[\omega]\right|$ for $10 \leq \omega \leq 600$

\begin{tabular}{cccccc}
\hline$\omega \backslash \mathrm{N}$ & 1 & 2 & 3 & 4 & 5 \\
\hline 10 & $2.70 \mathrm{E}-2$ & $3.58 \mathrm{E}-3$ & $5.00 \mathrm{E}-4$ & $2.20 \mathrm{E}-4$ & $1.13 \mathrm{E}-5$ \\
20 & $7.22 \mathrm{E}-3$ & $5.99 \mathrm{E}-4$ & $6.80 \mathrm{E}-5$ & $2.21 \mathrm{E}-5$ & $3.62 \mathrm{E}-7$ \\
30 & $3.34 \mathrm{E}-3$ & $1.84 \mathrm{E}-4$ & $1.74 \mathrm{E}-5$ & $4.11 \mathrm{E}-6$ & $3.08 \mathrm{E}-8$ \\
40 & $2.01 \mathrm{E}-3$ & $7.62 \mathrm{E}-5$ & $6.18 \mathrm{E}-6$ & $1.12 \mathrm{E}-6$ & $8.66 \mathrm{E}-9$ \\
50 & $1.30 \mathrm{E}-3$ & $3.89 \mathrm{E}-5$ & $2.68 \mathrm{E}-6$ & $3.93 \mathrm{E}-7$ & $3.22 \mathrm{E}-9$ \\
100 & $3.17 \mathrm{E}-4$ & $4.90 \mathrm{E}-6$ & $1.82 \mathrm{E}-7$ & $1.34 \mathrm{E}-8$ & $8.50 \mathrm{E}-11$ \\
150 & $1.37 \mathrm{E}-4$ & $1.47 \mathrm{E}-6$ & $3.65 \mathrm{E}-8$ & $1.80 \mathrm{E}-9$ & $8.25 \mathrm{E}-12$ \\
200 & $7.59 \mathrm{E}-5$ & $6.23 \mathrm{E}-7$ & $1.16 \mathrm{E}-8$ & $4.28 \mathrm{E}-10$ & $1.52 \mathrm{E}-12$ \\
250 & $4.93 \mathrm{E}-5$ & $3.17 \mathrm{E}-7$ & $4.77 \mathrm{E}-9$ & $1.41 \mathrm{E}-10$ & $4.06 \mathrm{E}-13$ \\
300 & $3.53 \mathrm{E}-5$ & $1.81 \mathrm{E}-7$ & $2.30 \mathrm{E}-9$ & $5.66 \mathrm{E}-11$ & $1.37 \mathrm{E}-13$ \\
350 & $2.66 \mathrm{E}-5$ & $1.13 \mathrm{E}-7$ & $1.24 \mathrm{E}-9$ & $2.62 \mathrm{E}-11$ & $5.46 \mathrm{E}-14$ \\
400 & $2.06 \mathrm{E}-5$ & $7.50 \mathrm{E}-8$ & $7.30 \mathrm{E}-10$ & $1.34 \mathrm{E}-11$ & $2.46 \mathrm{E}-14$ \\
450 & $1.61 \mathrm{E}-5$ & $5.30 \mathrm{E}-8$ & $4.56 \mathrm{E}-10$ & $7.46 \mathrm{E}-12$ & $1.22 \mathrm{E}-14$ \\
500 & $1.26 \mathrm{E}-5$ & $3.92 \mathrm{E}-8$ & $2.99 \mathrm{E}-10$ & $4.41 \mathrm{E}-12$ & $6.48 \mathrm{E}-15$ \\
550 & $1.01 \mathrm{E}-5$ & $2.98 \mathrm{E}-8$ & $2.04 \mathrm{E}-10$ & $2.74 \mathrm{E}-12$ & $3.66 \mathrm{E}-15$ \\
600 & $8.44 \mathrm{E}-6$ & $2.30 \mathrm{E}-8$ & $1.44 \mathrm{E}-10$ & $1.77 \mathrm{E}-12$ & $2.17 \mathrm{E}-15$ \\
\hline
\end{tabular}

Example 2 In our second integral

$$
\mathcal{I}[\omega]=\int_{0}^{1} \frac{1}{x+1} e^{i \omega\left(x^{2}+x+1\right)^{1 / 3}} d x
$$

$f(x)=\frac{1}{x+1}$ and $g(x)=\left(x^{2}+x+1\right)^{1 / 3}$ are not polynomials. So in order to apply Algorithm TQ(N), we replace, according to (18)-(19), $f(x)$ and $g^{\prime}(x)$ by $\tilde{f}(x)$ and $\tilde{g}^{\prime}(x)$ that represent their respective $\mathrm{N} t h$ truncated two-point Taylor series expansions at $x=0$ and $x=1$.

We computed $\mathcal{I}_{N}[\omega]$ by means of Algorithm TQ(N) with $N=1,2,3,4,5$ and different values of frequencies $\omega$. The errors $\left|\mathcal{E}_{N}[\omega]\right|$ are displayed in Table 2 for some values of $\omega$ and Figure 3-right shows the scaled error $\omega^{2}\left|\mathcal{E}_{1}[\omega]\right|$. Again, asymptotically we have $\mathcal{E}_{1}[\omega]=O\left(\omega^{-2}\right)$.

The calculations were repeated for large frequencies $\omega$ where $4000 \leq \omega \leq 16000$ : the absolute errors are summarized in Table 3 and a plot of the scaled error $\omega^{2}\left|\mathcal{E}_{1}[\omega]\right|$ for $\omega=4000,4020,4040, \ldots, 16000$ is shown in Figure 3-left. The latter conforms that our results conserve the asymptotic property even when very large frequencies are considered. 

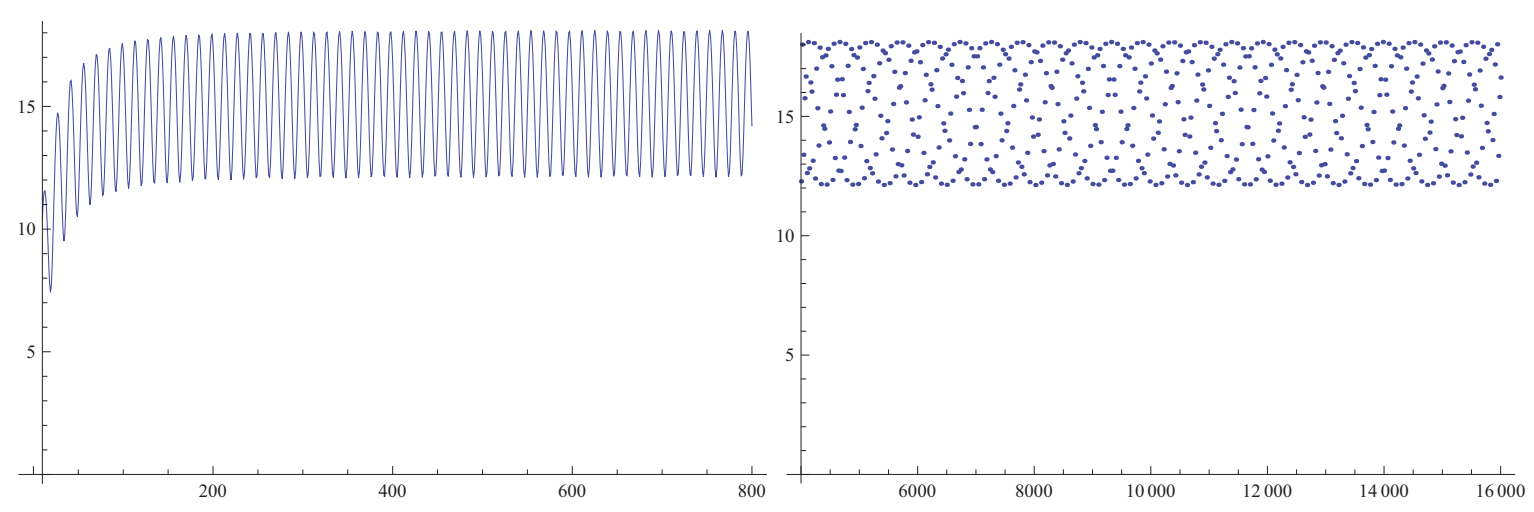

Figure 3 (Example 2). Plot of the scaled error $\omega^{2}\left|\mathcal{E}_{1}[\omega]\right|$ for $40 \leq \omega \leq 800$ [left] and for $\omega=4000,4020,4040, \ldots, 16000$ [right]

Table 2 (Example 2). Integral (25) is approximated by TQ(N), $N=1,2,3,4,5$. List of $\left|\mathcal{E}_{N}[\omega]\right|$ for $40 \leq \omega \leq 1000$

\begin{tabular}{cccccc}
\hline$\omega \backslash \mathrm{N}$ & 1 & 2 & 3 & 4 & 5 \\
\hline 40 & $9.67 \mathrm{E}-3$ & $1.24 \mathrm{E}-3$ & $3.89 \mathrm{E}-4$ & $8.68 \mathrm{E}-5$ & $1.56 \mathrm{E}-5$ \\
100 & $1.73 \mathrm{E}-3$ & $1.17 \mathrm{E}-4$ & $1.59 \mathrm{E}-5$ & $2.13 \mathrm{E}-6$ & $3.11 \mathrm{E}-7$ \\
160 & $5.67 \mathrm{E}-4$ & $3.64 \mathrm{E}-5$ & $2.39 \mathrm{E}-6$ & $2.61 \mathrm{E}-7$ & $2.54 \mathrm{E}-8$ \\
220 & $2.48 \mathrm{E}-4$ & $1.53 \mathrm{E}-5$ & $6.70 \mathrm{E}-7$ & $5.67 \mathrm{E}-8$ & $4.40 \mathrm{E}-9$ \\
280 & $1.89 \mathrm{E}-4$ & $6.63 \mathrm{E}-6$ & $2.96 \mathrm{E}-7$ & $1.61 \mathrm{E}-8$ & $1.17 \mathrm{E}-9$ \\
340 & $1.55 \mathrm{E}-4$ & $3.11 \mathrm{E}-6$ & $1.50 \mathrm{E}-7$ & $5.85 \mathrm{E}-9$ & $3.85 \mathrm{E}-10$ \\
400 & $1.05 \mathrm{E}-4$ & $2.11 \mathrm{E}-6$ & $7.39 \mathrm{E}-8$ & $2.82 \mathrm{E}-9$ & $1.41 \mathrm{E}-10$ \\
460 & $6.13 \mathrm{E}-5$ & $1.66 \mathrm{E}-6$ & $3.69 \mathrm{E}-8$ & $1.53 \mathrm{E}-9$ & $5.82 \mathrm{E}-11$ \\
520 & $4.77 \mathrm{E}-5$ & $1.14 \mathrm{E}-6$ & $2.32 \mathrm{E}-8$ & $8.13 \mathrm{E}-10$ & $2.90 \mathrm{E}-11$ \\
580 & $4.98 \mathrm{E}-5$ & $6.85 \mathrm{E}-7$ & $1.73 \mathrm{E}-8$ & $4.28 \mathrm{E}-10$ & $1.62 \mathrm{E}-11$ \\
640 & $4.38 \mathrm{E}-5$ & $4.73 \mathrm{E}-7$ & $1.20 \mathrm{E}-8$ & $2.59 \mathrm{E}-10$ & $9.00 \mathrm{E}-12$ \\
700 & $3.04 \mathrm{E}-5$ & $4.38 \mathrm{E}-7$ & $7.45 \mathrm{E}-9$ & $1.82 \mathrm{E}-10$ & $4.94 \mathrm{E}-12$ \\
760 & $2.10 \mathrm{E}-5$ & $3.78 \mathrm{E}-7$ & $4.90 \mathrm{E}-9$ & $1.26 \mathrm{E}-10$ & $2.94 \mathrm{E}-12$ \\
820 & $2.21 \mathrm{E}-5$ & $2.70 \mathrm{E}-7$ & $4.05 \mathrm{E}-9$ & $8.08 \mathrm{E}-11$ & $1.98 \mathrm{E}-12$ \\
880 & $2.32 \mathrm{E}-5$ & $1.81 \mathrm{E}-7$ & $3.39 \mathrm{E}-9$ & $5.23 \mathrm{E}-11$ & $1.36 \mathrm{E}-12$ \\
940 & $1.90 \mathrm{E}-5$ & $1.63 \mathrm{E}-7$ & $2.47 \mathrm{E}-9$ & $3.97 \mathrm{E}-11$ & $8.84 \mathrm{E}-13$ \\
1000 & $1.29 \mathrm{E}-5$ & $1.62 \mathrm{E}-7$ & $1.68 \mathrm{E}-9$ & $3.19 \mathrm{E}-11$ & $5.72 \mathrm{E}-13$ \\
\hline
\end{tabular}

Table 3 (Example 2). Integral (25) is approximated by TQ(N), $N=1,2,3,4,5$. List of $\left|\mathcal{E}_{N}[\omega]\right|$ for $4000 \leq \omega \leq$ 16000

\begin{tabular}{cccccc}
\hline$\omega \backslash \mathrm{N}$ & 1 & 2 & 3 & 4 & 5 \\
\hline 4000 & $7.67 \mathrm{E}-7$ & $2.59 \mathrm{E}-9$ & $6.45 \mathrm{E}-12$ & $3.15 \mathrm{E}-14$ & $1.40 \mathrm{E}-16$ \\
5000 & $7.15 \mathrm{E}-7$ & $9.98 \mathrm{E}-10$ & $3.24 \mathrm{E}-12$ & $8.947 \mathrm{E}-15$ & $4.07 \mathrm{E}-17$ \\
6000 & $3.93 \mathrm{E}-7$ & $7.17 \mathrm{E}-10$ & $1.36 \mathrm{E}-12$ & $4.00 \mathrm{E}-15$ & $1.27 \mathrm{E}-17$ \\
7000 & $2.97 \mathrm{E}-7$ & $4.43 \mathrm{E}-10$ & $7.49 \mathrm{E}-13$ & $1.83 \mathrm{E}-15$ & $5.08 \mathrm{E}-18$ \\
8000 & $2.77 \mathrm{E}-7$ & $2.47 \mathrm{E}-10$ & $4.92 \mathrm{E}-13$ & $8.60 \mathrm{E}-16$ & $2.41 \mathrm{E}-18$ \\
9000 & $1.502 \mathrm{E}-7$ & $2.28 \mathrm{E}-10$ & $2.50 \mathrm{E}-13$ & $5.48 \mathrm{E}-16$ & $1.08 \mathrm{E}-18$ \\
10000 & $1.72 \mathrm{E}-7$ & $1.31 \mathrm{E}-10$ & $1.98 \mathrm{E}-13$ & $2.86 \mathrm{E}-16$ & $6.28 \mathrm{E}-19$ \\
11000 & $1.27 \mathrm{E}-7$ & $1.10 \mathrm{E}-10$ & $1.27 \mathrm{E}-13$ & $1.87 \mathrm{E}-16$ & $3.42 \mathrm{E}-19$ \\
12000 & $9.25 \mathrm{E}-8$ & $9.25 \mathrm{E}-11$ & $8.28 \mathrm{E}-14$ & $1.27 \mathrm{E}-16$ & $1.96 \mathrm{E}-19$ \\
13000 & $1.07 \mathrm{E}-7$ & $5.59 \mathrm{E}-11$ & $7.15 \mathrm{E}-14$ & $7.48 \mathrm{E}-17$ & $1.32 \mathrm{E}-19$ \\
14000 & $6.49 \mathrm{E}-8$ & $5.95 \mathrm{E}-11$ & $4.37 \mathrm{E}-14$ & $5.95 \mathrm{E}-17$ & $7.69 \mathrm{E}-20$ \\
15000 & $7.19 \mathrm{E}-8$ & $4.15 \mathrm{E}-11$ & $3.77 \mathrm{E}-14$ & $3.89 \mathrm{E}-17$ & $5.40 \mathrm{E}-20$ \\
16000 & $6.49 \mathrm{E}-8$ & $3.33 \mathrm{E}-11$ & $2.95 \mathrm{E}-14$ & $2.78 \mathrm{E}-17$ & $3.69 \mathrm{E}-20$ \\
\hline
\end{tabular}


Example 3 We consider now the multivariate oscillatory integral

$$
\mathcal{I}[\omega]=\int_{0}^{\frac{3}{2}} \int_{0}^{\sqrt{1-y^{2}}}\left(e^{x y}+y^{2}+1\right) e^{i \omega\left(\cos \left(\frac{y}{2}+1\right)+x^{2}+x\right)} d x d y .
$$

As explained in section 4, we apply TQ(N) twice: First we integrate with respect to $x$ and we get a new integrand as a function of $y$. Repeating the same algorithm to this new function gives an approximate value for the required integral $\mathcal{I}_{N}[\omega]$. Table 4 shows the absolute value of the error $\left|\mathcal{E}_{N}[\omega]\right|$ for some values of $\omega$. Figure 4 shows the scaled error $\omega^{N+2}\left|\mathcal{E}_{N}[\omega]\right|$ for $N=1$ (down) and $N=2$ (up). Here $50 \leq \omega \leq 10000$.

Example 4 Our last example is integrated on the unit semi-disk,

$$
\mathcal{I}[\omega]=\int_{-1}^{1} \int_{-\sqrt{1-y^{2}}}^{\sqrt{1-y^{2}}} \cos y e^{i \omega\left(x^{2}+y^{2}+3 x+4 y\right)} d x d y .
$$

Again, as in the previous example, we apply the algorithm presented in Section 4 for multivariate integrals. The exact values of $\left|\mathcal{E}_{N}[\omega]\right|$ where $500 \leq \omega \leq 20000$ and $N=1,2$ are recorded in Table 4-right. In Figure 4-right we draw the scaled error $\omega^{N+2}\left|\mathcal{E}_{N}[\omega]\right|$ where $N=1$ and $N=2$.
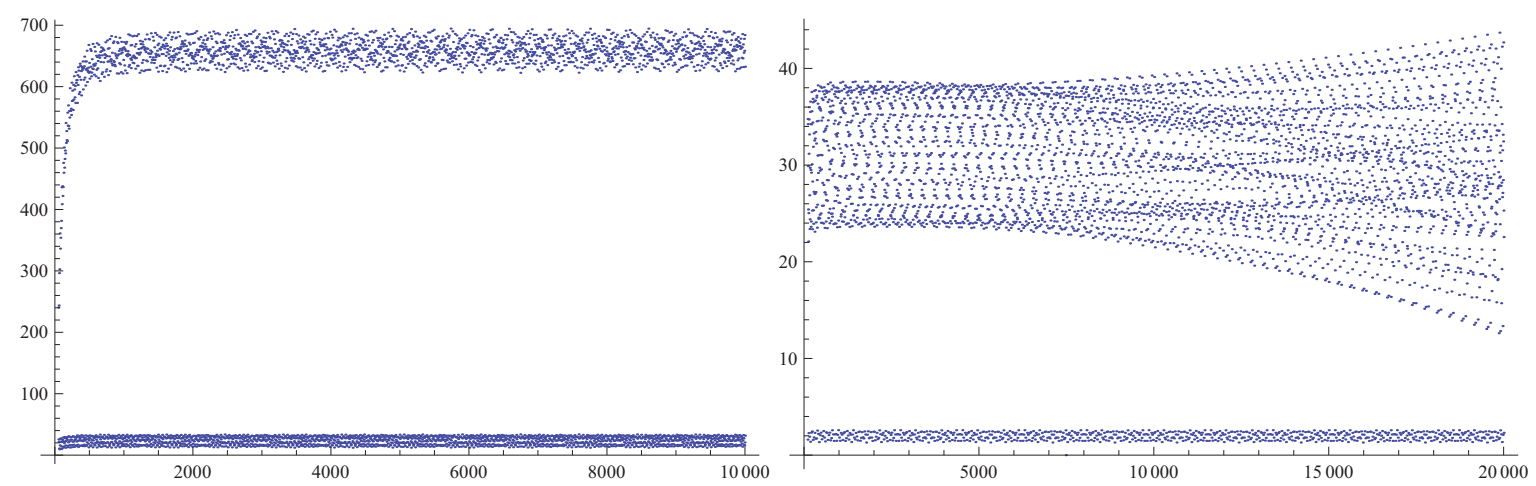

Figure 4. [left] (Example 3). Plot of the scaled error $\omega^{3}\left|\mathcal{E}_{1}[\omega]\right|$ (up) and $\omega^{4}\left|\mathcal{E}_{2}[\omega]\right|$ (down) where $50 \leq \omega \leq 10000$. [right] (Example 4). Plot of the scaled error $\omega^{3}\left|\mathcal{E}_{1}[\omega]\right|$ (up) and $\omega^{4}\left|\mathcal{E}_{2}[\omega]\right|$ (down) where $100 \leq \omega \leq 20000$ 
Table 4 (Example 3). [left] Integral (26) is approximated by TQ(N), $N=1,2$. List of $\left|\mathcal{E}_{N}[\omega]\right|$ for $100 \leq \omega \leq 10^{4}$. [right] (Example 4). Integral (27) is approximated by TQ(N), $N=1,2$. List of $\left|\mathcal{E}_{N}[\omega]\right|$ for $500 \leq \omega \leq 20000$

\begin{tabular}{ccc}
\hline$\omega \backslash N$ & 1 & 2 \\
\hline 100 & $2.177 \mathrm{E}-5$ & $4.086 \mathrm{E}-6$ \\
500 & $2.567 \mathrm{E}-7$ & $9.808 \mathrm{E}-9$ \\
1000 & $1.761 \mathrm{E}-8$ & $6.724 \mathrm{E}-10$ \\
1500 & $7.518 \mathrm{E}-9$ & $1.324 \mathrm{E}-10$ \\
2000 & $2.954 \mathrm{E}-9$ & $4.194 \mathrm{E}-11$ \\
2500 & $1.301 \mathrm{E}-9$ & $1.700 \mathrm{E}-11$ \\
3000 & $1.179 \mathrm{E}-9$ & $7.793 \mathrm{E}-12$ \\
3500 & $3.556 \mathrm{E}-10$ & $4.386 \mathrm{E}-12$ \\
4000 & $4.906 \mathrm{E}-10$ & $2.519 \mathrm{E}-12$ \\
4500 & $1.836 \mathrm{E}-10$ & $1.669 \mathrm{E}-12$ \\
5000 & $2.120 \mathrm{E}-10$ & $1.070 \mathrm{E}-12$ \\
5500 & $1.452 \mathrm{E}-10$ & $7.221 \mathrm{E}-13$ \\
6000 & $1.039 \mathrm{E}-10$ & $5.020 \mathrm{E}-13$ \\
6500 & $1.169 \mathrm{E}-10$ & $3.516 \mathrm{E}-13$ \\
7000 & $4.367 \mathrm{E}-11$ & $2.767 \mathrm{E}-13$ \\
7500 & $7.235 \mathrm{E}-11$ & $2.073 \mathrm{E}-13$ \\
8000 & $2.839 \mathrm{E}-11$ & $1.691 \mathrm{E}-13$ \\
8500 & $4.437 \mathrm{E}-11$ & $1.278 \mathrm{E}-13$ \\
9000 & $3.234 \mathrm{E}-11$ & $1.001 \mathrm{E}-13$ \\
9500 & $2.692 \mathrm{E}-11$ & $7.926 \mathrm{E}-14$ \\
10000 & $3.168 \mathrm{E}-11$ & $6.324 \mathrm{E}-14$ \\
\hline
\end{tabular}

\begin{tabular}{ccc}
\hline$\omega \backslash \mathrm{N}$ & 1 & 2 \\
\hline 500 & $1.313 \mathrm{E}-8$ & $5.905 \mathrm{E}-10$ \\
1000 & $1.819 \mathrm{E}-9$ & $3.439 \mathrm{E}-11$ \\
2000 & $3.017 \mathrm{E}-10$ & $1.750 \mathrm{E}-12$ \\
3000 & $9.244 \mathrm{E}-11$ & $2.956 \mathrm{E}-13$ \\
4000 & $3.279 \mathrm{E}-11$ & $1.105 \mathrm{E}-13$ \\
5000 & $1.310 \mathrm{E}-11$ & $5.702 \mathrm{E}-14$ \\
6000 & $7.285 \mathrm{E}-12$ & $2.978 \mathrm{E}-14$ \\
7000 & $5.371 \mathrm{E}-12$ & $1.447 \mathrm{E}-14$ \\
8000 & $4.234 \mathrm{E}-12$ & $6.563 \mathrm{E}-15$ \\
9000 & $3.264 \mathrm{E}-12$ & $3.618 \mathrm{E}-15$ \\
10000 & $2.277 \mathrm{E}-12$ & $2.973 \mathrm{E}-15$ \\
11000 & $1.341 \mathrm{E}-12$ & $2.483 \mathrm{E}-15$ \\
12000 & $7.836 \mathrm{E}-13$ & $1.823 \mathrm{E}-15$ \\
13000 & $8.140 \mathrm{E}-13$ & $1.174 \mathrm{E}-15$ \\
14000 & $8.882 \mathrm{E}-13$ & $6.981 \mathrm{E}-16$ \\
15000 & $7.631 \mathrm{E}-13$ & $4.832 \mathrm{E}-16$ \\
16000 & $5.153 \mathrm{E}-13$ & $4.527 \mathrm{E}-16$ \\
17000 & $3.037 \mathrm{E}-13$ & $4.329 \mathrm{E}-16$ \\
18000 & $2.641 \mathrm{E}-13$ & $3.631 \mathrm{E}-16$ \\
19000 & $2.952 \mathrm{E}-13$ & $2.591 \mathrm{E}-16$ \\
20000 & $2.883 \mathrm{E}-13$ & $1.629 \mathrm{E}-16$ \\
\hline
\end{tabular}

\section{Conclusion}

We have presented an approach to evaluate highly oscillatory integrals of the form (1) and (23). The method applies to integrals involving moderate frequencies of different sizes and the accuracy of the method improves when the frequency increases. With our procedures the integral is obtained in terms of the value of the function and its derivatives at the boundary points. The order of our method is $O\left(\omega^{-N-1}\right)$ for univariate and $O\left(\omega^{-N-d}\right)$ if the domain of the integral is $N$ dimensional.

\section{Acknowledgments}

The author appreciates a financial support from the Public Authority for Applied Education and Training in Kuwait.

\section{References}

Abramowitz, M., \& Stegun, I. A. (1965). Handbook of mathematical functions with formulas, graphs, and mathematical tables. New York, NY: Dover Publications.

Davis, P. J. (1975). Interpolation and Approximation. New York, NY: Dover Publications.

Davis, P. J., \& Rabinowitz, P. (1980). Methods of numerical integration (2nd ed.). Orlando, FL: Academic Press.

Filon, L. N. G. (1928). On the quadrature formula for trigonometric integrals. Proc. Roy. Soc. Edinburgh, 49, $38-47$.

Huybrechs, D., \& Vandewalle, S. (2006). On the evaluation of highly oscillatory integrals by analytic continuation. SIAM J. Numer. Anal., 44, 1026-1048. http://dx.doi.org/10.1137/050636814

Iserles, A. (2004). On the method of Neumann series for highly oscillatory equations. BIT, 44, 473-488. http://dx.doi.org/10.1023/B:BITN.0000046810.25353.95

Iserles, A., \& Nørsett, S. P. (2005). Efficient quadrature of highly oscillatory integrals using derivatives. Proc. $R$. Soc. A, 461, 1383-1399. http://dx.doi.org/10.1098/rspa.2004.1401

Iserles, A., \& Nørsett, S. P. (2006). Quadrature methods for multivariate highly oscillatory integrals using derivatives. Math. Comp., 75, 1233-1258. http://dx.doi.org/10.1090/S0025-5718-06-01854-0

Ixaru, L. Gr., \& Vanden Berghe, G. (2004). Exponential Fitting. Dordrecht: Kluwer Academic Publishers. 
Levin, D. (1996). Fast integration of rapidly oscillatory functions. J. Comput. App. Math., 67, 95-101. http://dx.doi.org/10.1016/0377-0427(94)00118-9

Luke, Y. L. (1954). On the computation of oscillatory integrals. Proc. Cambridge Phil. Soc., 50, 269-277.

Olver, S. (2006). On the quadrature of multivariate highly oscillatory integrals over non-polytope domains. Numer. Math., 4(103), 643-665. http://dx.doi.org/10.1007/s00211-006-0009-2

Ortiz, E. L. (1969). The Tau Method. SIAM J. Numer. Anal., 6, 480-492. http://dx.doi.org/10.1137/0706044

Stein, S. (1993). Harmonic Analysis: Real-Valued Methods, Orthogonality and Oscillatory Integrals. Princeton, NJ: Princeton University Press.

Van Daele, M., Vanden Berghe, G., \& Vande Vyver, H. (2005). Exponentially fitted quadrature rules of Gauss type for oscillatory integrands. Applied Numerical Mathematics, 53(2-4), 509-526. http://dx.doi.org/10.1016/j.apnum.2004.08.018

\section{Copyrights}

Copyright for this article is retained by the author(s), with first publication rights granted to the journal.

This is an open-access article distributed under the terms and conditions of the Creative Commons Attribution license (http://creativecommons.org/licenses/by/3.0/). 